\title{
The development of p53 tumor diagnosis and drug design strategy in its disordered region
}

\author{
Yiyun Zhang \\ China Medical University, Liaoning Shenyang, China
}

\begin{abstract}
As a tumor suppressor protein, p53 is directly involved in the control of cell cycle arrest, which plays a crucial role in cell metabolism, growth, apoptosis and responses to different types of damage or stress processes. And since the tumor and cancer are usually related to the mutation or functional inactivation of the p53, it's necessary to investigate the mechanism of p53 functioning the tumor and other disease, trying to figure out its drug design strategy. In this paper, a brief introduction of p53 is given, including the structure, function and properties. Subsequently, the relationship of p53 gene and cancer is also discussed. In the end, a new rational drug-design for disordered region of p53 was summarized
\end{abstract}

\section{INTRODUCTION}

The tumor suppressor protein p53 participates in a variety of signal transduction processes inside/outside the cell microenvironment, and it regulates gene expression, cell metabolism, growth, cell cycle arrest, apoptosis and senescence and other cell physiological activities [1,2]. MDM2 and MDMX are two important regulators of $\mathrm{p} 53$, and they interact with $\mathrm{p} 53$ to regulate p53 expression and translational activity [1-3].

P53 is a transcription factor involved in cell cycle reg-ulation, apoptosis and many other life processes. Since p53 can inhibit tumors through the apoptotic pathway, cancer scientists have deeply studied the interaction between p53 and MDM2. The sequence of p53 can be divided into three parts: N-terminal domain, DNA binding domain (DBD) and C-terminal domain. Among them, the DNA binding domain is completely folded, and both ends of the domain are naturally disordered $[4,5]$.

The function of $\mathrm{p} 53$ relies on its structural conformation, with the core domain responsible for specific DNA binding, and the expression of p53 under control of a complex network of transcriptional and posttranscriptional regulation. There are many transcription factor and metabolic product that may post an effect upon the p53 regulation, and the exact mechanism of the p53 regulation remain unclear. Tumor cells of-ten involve mutated p53 or/and the mechanism of inactivation of $\mathrm{p} 53$, such a differences between lesion tissue and normal cells provide a way to tumor diagnose. And the reactivation and gene therapy is research hotpot when it comes to anti-cancer drug invention.

\section{THE CHARACTERS PROPERTIES OF P53}

\subsection{P53 protein}

P53 is considered as one of the most important regulatory factors in many signal pathway regulatory proteins in cells. As a sequence-specific transcription factor, p53 can mediate transcriptional activation or inhibition under different conditions [6]. The p53 protein was originally discovered in 1979 when investigating human family adenomatous polyposis, mainly dispersed in the nucleus pulp, working as "guardian of the genome".

As a tumor suppressor gene, p53 can code protein known as transcriptional factor, plays an important role in $\mathrm{G} 1$ checkpoint, halts cell division if DNA damages are detected and stimulates the activity of repair enzymes at the same time. When the DNA damage is beyond repair, the p53 protein can trigger the destruction of the cell.

Mutation in p53 gene may lead to tumor when the structure of the protein altered. The conformation changes can result in loss of ability of DNA-binding, apoptosis, cell division arrest stimulation. Thus the p53 protein is closely related with the tumor and cancer.

\subsection{Structure of p53 protein}

The gene encoding the $\mathrm{p} 53$ protein is located on the short arm of human chromosome 17 , and contains 11 exons. The native p53 protein transcribed and translated consists of 393 amino acids with a protein molecular weight of $53 \mathrm{kD}$. The half-life of p53 protein in normal cells is $6-20 \mathrm{~min}$.

The p53 protein is a tetramer in shape, with four main functional domains. The N-terminal region, which is intrinsically disordered, consists of the transactivation domain (TAD). TF11D works on such region by binding with the TATA box in the promotor and activate the

\footnotetext{
* Corresponding author: robbieswan@163.com
} 
transcription. TAD can be subdivided to TAD1 and TAD2 (residue 1-61), and the $\alpha$ helix structure in those regions is also the main target site of MDM2. There is a down-regulator of p53 gene which can transport p53 protein from nucleus to cytosol, which having highly expression at tumor cells. Followed the D-terminal region is the proline-rich region contains with five PXXP motifs. Located in the p53 conversed core domain is the highly specific DNA-binding domain responsible for the sequence-specific DNA binding. Finally, there is the Cterminal domain, consists of NLS and NES, binding with the DNA non-highly-specifically or with other protein, it can also be subject to posttranslational modification and p53 functional regulation. The tetramerization domain (TD) lies between the $\mathrm{C}$ terminal region and DNAbinding domain. [7]

\subsection{Function of p53 protein}

The main function of $\mathrm{p} 53$ protein is to supervise the DNA damage and abort cell division if necessary, acts like transcriptional activator. There are two checkpoints in which p53 protein works. Firstly, at G1 checkpoint, $\mathrm{p} 53$ protein prevent the cell division from entering the $\mathrm{S}$ phase and repair the damage. It promotes expression of p21 protein, which inhibit the cyclin-E and promotes cell division arrest. At the same time, the proteins regarding the DNA repair, such as $\mathrm{Ku} 70$, RNR, are expressed. Secondly, more than one pathway take part in the network of p53 protein in the effect of inhibiting cell division at $\mathrm{G} 2$ checkpoint.

When the damage in gene is too severe to repair, p53 protein promotes the apoptosis of the cell with its upregulate factors like BAX, PUMA, NOXA. Some genes are specifically activated by 553 protein, for example WAF1/CIPI may inhibit the activity of the CDK complexes which is important for cell cycle progress. P21 can prevent the phosphorylation of $\mathrm{pRB}$, a protein binding to E2F and preventing its activating CDK2. Moreover, wild-type p53 transactivates the GADD45 gene, initiating the DNA repair process. In addition, via intrinsic pathway of apoptosis, it promotes the release of cytochrome $\mathrm{C}$ from mitochondria, activates a series of cleavage cascade, and finally leads to cell death. Other function, like Anti-angiogenesis in tumor tissue, promotes cell senescence in mammal and metabolic effect. [8]

\section{THE RELATIONSHIP BETWEEN P53 GENE AND CANCER}

Mutation or functional inactivation of the tumor suppressor p53 often leads to cancer. Mutations in the TP53 gene encoding the p53 protein occur in approximately half of the tumor samples. Gene mutations are the main way to cause the loss of normal function of $\mathrm{p} 53$.

A large number of mutations occur in the core domain, frequents are relatively high at residues site 248 and 273 . Some mutations only have minor effect on the overall conformation of the p53 protein, while some missense mutation, which is predominate in the mutation of p53 gene, can lead to structure change of the protein and causative functional inactivation.

Inactivation of p53 often prevalent in tumorigenesis, caused by structural mutations that alter the overall structure of p53 protein that may lead to the thermodynamic instability of $\mathrm{p} 53$ protein at body temperature, and/or contact mutation that impair DNA binding ability so does the ability to activate relevant genes. Many mutation occur in the core domain like $\beta$ Sandwich mutation and the mutation that alter zinic ligand. The mutation can be accumulated.

Tumor viruses involves in about $15 \%$ of total cancer cases, they encode protein which can binding with p53 or induce degradation, interrupting the normal control of cell cycle for the sake of viruses replication.

The upstream or downstream pathway of effect of p53 can also perturb its control over cell cycle and lead to tumor. The regulation of p53 is directly under control of expression of regulator factor MDM2 and MDMX. At least 72 MDM2 splice variants had been described and some of them associated with tumor, commonly variants like MDM2-a,b,c are found in tumor tissue.

\section{APPLICATION OF P53 IN TUMOR DIAGNOSIS}

According to the type differences of p53 gene mutation, we may distinct different cancer. In tumor tissue, the $\mathrm{Rb}$ and p53 protein structure may change due to mutation and can be detect by immune reaction. The transcription product of $\mathrm{Rb}$ and $\mathrm{p} 53$ from lesion tissue can be determined by radioisotope or immunological methods.

MDM2 antagonists acts as an anticancer drug by binding with NDM2 replace of p53 competitively, interrupting the down-regulating process and increase the expression of p53. Another way is to reactivate mutated p53 protein. Some Drug like Phikan083 have the chemical structure that can mend the crevice caused by mutation (e.g. Y220C) give the protein a normal surface structure and reactivate it. Some small molecular compound or polypeptide all have such effect.

As the regulation of $\mathrm{p} 53$ is a complicated signal network, buy interfering the up/down stream pathway, the effect of the gene can be altered. For one example, transcription product of MDM2 can have positive regulation of MDM2 gene, for another, some polysaccharides can regulate the protein affecting p53 express. PLK1 ( polo-like kinasel) promotes the process of mitosis and negatively regulates the function of p53 by binding to p53 gene and inhibit its expression. As a result, developing PLK1 inhibitor provides a way to increase apoptosis to treat tumor. Transcription factor like Snaill can bind to p53 to form complex that make p53 prone to degradation

\section{DRUG DESIGN FOR DISORDERED REGION OF P53}


Tumor suppressor protein p53 is involved in cell cycle control, senescence and response to apoptosis or other cellular stress signals. The p53 protein is regulated by binding to multiple targets such as MDM2 and Taz2 [9]. Small molecules have been screened to inhibit the p53MDM2 interaction and reactivate the p53 pathway in cancer cells. [10]

These small molecules make difference by binding to MDM2 in the p53 binding pocket but do not directly interact with p53. Therefore, this is a drug design example related to disordered region of $\mathrm{p} 53$.

\section{CONCLUSION}

P53 regulates tissue proliferation and promotes the programmed cell death (apoptosis). This paper summarized the characters and properties of $\mathrm{p} 53$, especially the structure and function. And the application of p53 in tumor diagnosis was proposed. What's more, the recent attempt of rational drug-design for p53 disordered region was given. Nearly half of tumors have p53 gene mutation, other may involve p53 inactivation or mechanism that bypass of its effect pathway. Better understanding about p53 may be facilitated to cancer diagnosis and drug development.

\section{References}

1. $\mathrm{Y}$ Barak, $\mathrm{T}$ Juven, $\mathrm{R}$ Haffner, M Oren. MDM2 expression is induced by wild type p53 activity [J]. Embo Journal, 1993, 12 (2): 461.

2. M Wade, YC Li, GM Wahl. MDM2, MDMX and p53 in oncogenesis and cancer therapy $[\mathrm{J}]$. Nature Reviews Cancer, 2013, 13 (2): 83-96.

3. JP Kruse, W Gu. Modes of p53 regulation [J]. Cell, 2009, 137 (4): 609-622.

4. VN Uversky, CJ Oldfield, AK Dunker. Intrinsically disordered proteins in human diseases: introducing the D2 concept [J]. Annual Review of Biophysics, 2008, 37 (1): 215.

5. LM Iakoucheva, CJ Brown, JD Lawson, Z Obradović, AK Dunker. Intrinsic disorder in cellsignaling and cancer-associated proteins $[\mathrm{J}]$. Journal of Molecular Biology, 2002, 323 (3): 573-584.

6. WD Funk, DT Pak, RH Karas, WE Wright, JW Shay. A transcriptionally active DNA-binding site for human p53 protein complexes [J]. Molecular \& Cellular Biology, 1992, 12 (6): 2866-2871.

7. AC Joerger, AR Fersht. Structural biology of the tumor suppressor p53 and cancer-associated mutants [J]. Annual Review of Biochemistry, 2008, 77 (1): 557-582.

8. WJ Lu, JF Amatruda, JM Abrams. P53 ancestry: gazing through an evolutionary lens [J]. Nature Reviews Cancer, 2009, 9 (10): 758-762.

9. Y Huang, Z Liu. Anchoring intrinsically disordered proteins to multiple targets: lessons from $\mathrm{N}$-terminus of the p53 protein [J]. International Journal of Molecular Sciences, 2011, 12 (2): 1410.
10. LT Vassilev, BT Vu, B Graves, D Carvajal, F Podlaski, Z Filipovic, N Kong, U Kammlott, C Lukacs, C Klein, N Fotouhi, EA Liu. In vivo activation of the p53 pathway by small-molecule antagonists of MDM2 [J]. Science, 2004, 303 (5659): 844-848. 